\title{
Pharmacologic manipulation of the respiratory control center in the infant
}

\author{
ROBERT G. LOCKE, DO \\ JOSEPH V. SALVIA, DO
}

The respiratory control center receives afferent stimuli from mechanical and neuromechanical sources. Information from both these sources, combined with voluntary and involuntary CNS control, effects stimulation of the respiratory muscles. In the infant, insufficiency of one or more of these elements of the respiratory control center is associated with considerable morbidity and mortality. Pharmacologic manipulation may provide a means of intervention. The xanthine derivative theophylline has been successfully used in the treatment of bronchopulmonary dysplasia and apnea in the infant. Naloxone, an endorphin antagonist, is widely used for the reversal of narcoticinduced respiratory depression but has not been shown to be clinically effective for either severely or moderately asphyxiated infants. Although doxapram has not been extensively studied and lacks an oral preparation, it is a potentially viable therapy in the treatment of refractory apnea and congenital hypoventilation syndromes. Almitrine's success in adults with chronic obstructive pulmonary disease has not been duplicated in infants with

From the Department of Pediatrics, University of Medicine and Dentistry of New Jersey-School of Osteopathic Medicine, Stratford, New Jersey.

Reprint requests to Robert G. Locke, DO, Department of Neonatology, St Christopher's Hospital for Children, Temple University School of Medicine, 2600 N Lawrence St, Philadelphia, PA 19133. similar respiratory impairments. Progesterone and prostaglandin, although proved to influence respiratory activity, should be regarded as very experimental therapeutic modalities.

The functions of the infant's respiratory control center and respiratory pump are to design a pattern of breathing that meets the infant's metabolic and ventilatory demands. Insufficiency of the respiratory system to meet these objectives may be associated with considerable morbidity and mortality. Pharmacologic manipulation of respiration may provide an adjuvant to the current methods of intervention. This review incorporates the current understanding of the physiology of the infant's respiratory control mechanisms with the potential for manipulation of those mechanisms by exogenous pharmacologic agents.

\section{Physiology of control}

The respiratory center receives afferent stimuli from mechanical and neuromechanical sources. These stimuli take into account the ventilatory and metabolic demands of the infant, resulting in a particular pattern of breathing. This situation is represented schematically in Figure 1.

\section{Chemoreceptors}

The peripheral oxygen-sensitive chemoreceptors consist of the aortic and carotid bodies. The carotid body is located at the bifurcation 


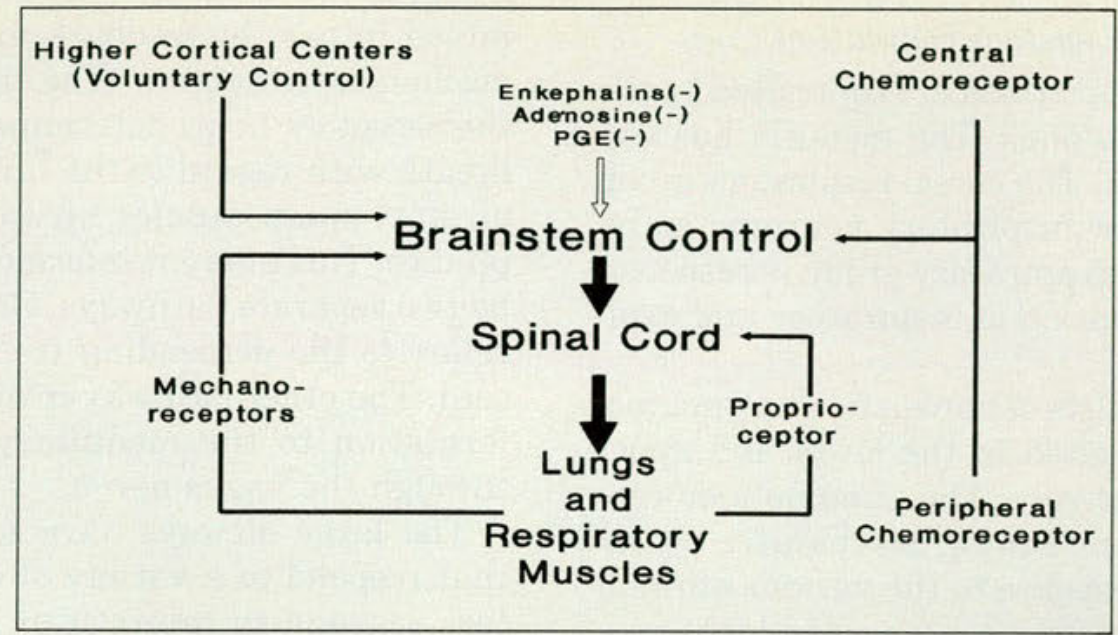

Figure. Physiology of the respiratory control center.

of the carotid artery. It receives a generous supply of blood and is one of the human body's most metabolically active sites. The neuromodulator-mediating carotid body activity at both the receptor and synapse levels is substance P. Antagonists to substance P block the peripheral and central hypoxic drives.

The carotid body responds to changes in oxygen tension and acid-base states. The primary response of the carotid body is to alterations in oxygen tension. However, approximately $30 \%$ of the ventilatory response to hypercapnea and nearly all the response to acute acidemias are stimulated by carotid body activity. Diminished oxygen tensions lead to increased efferent neuronal activity and stimulation of the respiratory center. This response is most pronounced at hypoxic levels of oxygen tension and is augmented by the presence of hypercapnea and acidemia. The stimulus to decrease the respiratory drive in the presence of hyperoxemia is negligible even in the presence of chronic carbon dioxide retention states. This differs from the situation in the adult, though the suppression of respiration in adult $\mathrm{CO}_{2}$ retainers on the basis of increased oxygen tension has been challenged.

Immediately after the infant's birth, the carotid body is inactive. This may be due to the fact that intrauterine $\mathrm{PaO}_{2}$ is usually $25 \mathrm{~mm}$ $\mathrm{Hg}$ to $28 \mathrm{~mm} \mathrm{Hg}$. Extrauterine tensions are relatively higher and do not elicit a response in the neonatal carotid body, even though a state of relative hypoxemia may exist. The re- setting of the carotid body occurs within the first few days of life. Persistent and profound hypoxemia does not necessarily result in a continued hyperventilatory response. This is probably due to tissue hypoxia of the respiratory muscles or of the respiratory center itself.

Very little is known about the aortic bodies in humans because ablation of the carotid body eliminates any detected peripheral response to oxygen tension or acid-base alterations in the blood. This contrasts with what has been discovered in several animal models in which the aortic bodies play a significant role in providing afferent information to the central respiratory control center. The reason for this difference is unclear.

It is not clear whether the central $\mathrm{CO}_{2}$ sensitive chemoreceptors are found in the ventral or dorsal respiratory groups or in a separate location within the medullary tissue. In contrast to the peripheral chemoreceptors, the central chemoreceptors are primarily responsive to $\mathrm{CO}_{2}$ and changes in the $\mathrm{pH}$ of the cerebrospinal fluid (CSF). There is a minimal response to oxygen tension. In the past, acetylcholine was considered to be the central chemoreceptor neuromodulator. Recent information suggests that aspartamine may be the more likely candidate or cocandidate. In contrast to the peripheral chemoreceptors, the central chemoreceptors are active at the time of birth, perhaps because intrauterine levels of $\mathrm{CO}_{2}$ are much closer to the extrauterine levels. 


\section{Central nervous system regulation}

Involuntary respirations are controlled by the medulla and the pons. The medulla has two centers of control. The dorsal respiratory group is responsible for inspiratory neuronal activity. The ventral respiratory group is responsible for a combination of inspiratory and expiratory activity.

The pons consists of apneustic and pneumotaxic centers located in the lower and upper portions, respectively. The pneumotaxic center acts as a fine-tuning mechanism by influencing the response to the various afferent stimuli. The apneustic center controls the neural activity associated with inspiration. There are extensive neuronal interconnections between these centers and the medullary dorsal and ventilatory tracts.

Voluntary influences from the cerebral cortex to the respiratory musculature descend through the spinal cord on tracts separate from those of the involuntary efferent stimuli. The voluntary influences from the cerebral cortex can alter the breathing pattern programmed by the medulla and the pons. This can produce a pattern either more or less advantageous to the infant.

The breathing pattern is also affected by different sleep states. In sleep stages 1 and 2, the lighter stages of sleep, the breathing pattern is irregular and periodic. Tidal volumes and respiratory rates vary for brief periods of time, creating short apneas and a periodic respiratory pattern. In stages 3 and 4 , the deeper stages of sleep, breathing is more regular and less varied.

Rapid eye movement (REM) sleep is characterized by markedly irregular breathing patterns, discoordinate chest wall motion, and relaxation of the smooth musculature of the airway causing increased resistance. The ventilatory response to hypercapnea is diminished in both REM and non-REM sleep, but the diminution is more pronounced in REM sleep. Arousal from sleep is preserved in response to hypercapnea unless significant hypoxia is present.

\section{Mechanical receptors}

A particular pattern of breathing is deter- mined in part by feedback mechanisms from mechanical receptors. The information from the receptors helps determine the design of a breath with regard to the function of the respiratory pump muscles, airway, and lung compliance. This afferent information is mediated by two separate pathways. One is a spinal arc reflex to the descending tracts in the spinal cord. The other pathway involves afferent information to the medullary control center through the vagus nerve.

The large airways have sensory receptors that respond to a variety of stimuli. J-receptors respond to interstitial lung fluid. Irritant receptors respond to mucosal irritation and stretch receptors sense lung volume-tension alterations. The vagus nerve carries the large airway mechanoreceptors' efferent output to the central control centers.

\section{Respiratory muscles}

The diaphragm provides the main inspiratory force of respiration. Its sole innervation is from the phrenic nerve. Contraction of the diaphragm causes downward displacement of the abdominal contents and outward displacement of the abdominal wall. The thoracic cage is expanded outward; the degree of expansion is inversely related to the degree of abdominal displacement.

The diaphragm consists of three fiber types: Type Ia fibers are slow twitch and high oxidative; type IIa fibers are fast oxidative and fatigue sensitive; and type IIb fibers are slow oxidative and fatigue resistant. Type I fibers are approximately three times more efficient than type II fibers and are therefore more resistant to fatigue. The relative composition of these fibers is age dependent. In preterm neonates, only $10 \%$ of the fibers are type I. In term infants this figure increases to $25 \%$. By two years of age and through adulthood, $50 \%$ of the fibers are type I. These histologic findings support clinical data demonstrating the disposition of the diaphragmatic fatigue present in infancy.

The efficiency of the diaphragmatic contraction is determined by the resting length of the muscle fibers. At relaxed fiber lengths, consistent with a dome-shaped diaphragm, the dia- 


\section{THE POWER TO EPADLCAIE

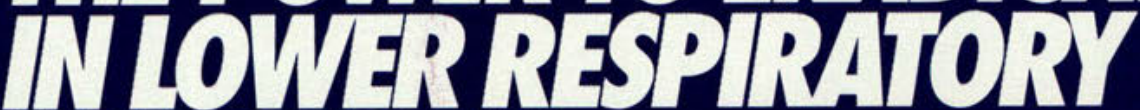 Hi:CIIOSS.}

Power that achieves $\mathbf{9 6} \%$ clinical success in lower respiratory infections including Pneumonia, Bronchitis, Bronchiectasis, and Lung Abscess. ${ }^{11 \dagger}$

NOTES: Concurrent administration of ciprofloxacin with theophylline may lead to elevated plasma concentrations of theophylline and prolongation of its elimination half-life. This may result in increased risk of theophylline-related adverse reactions. If concomitant use cannot be avoided, plasma levels of theophylline should be monitored and dosage adjustments made as appropriate. Antacids containing magnesium hydroxide or aluminum hydroxide interfere with the absorption of ciprofloxacin, resulting in serum and urine levels lower than desired; concurrent administration of these agents with ciprofloxacin should be avoided.

A history of hypersensitivity to ciprofloxacin is a contraindication to its use. A history of hypersensitivity to other quinolones may also contraindicate the use of ciprofloxacin.

CIPRO* SHOULD NOT BE USED IN CHILDREN, ADOLESCENTS, OR PREGNANT WOMEN.

-Achieves $96 \%$ favorable clinical response (resolution + improvement) of infections due to susceptible strains of indicated pathogens. See indicated organisms in prescribing information.

'Specific diagnoses of intections studied in clinical trials.'

' Clinical response data derived from a pool of studies conducted by investigators who may have used different criteria to define "resolution" plus "improvement."

See bnet summary of prescribing intormation and reterence on next page
Parenteral power in oral form

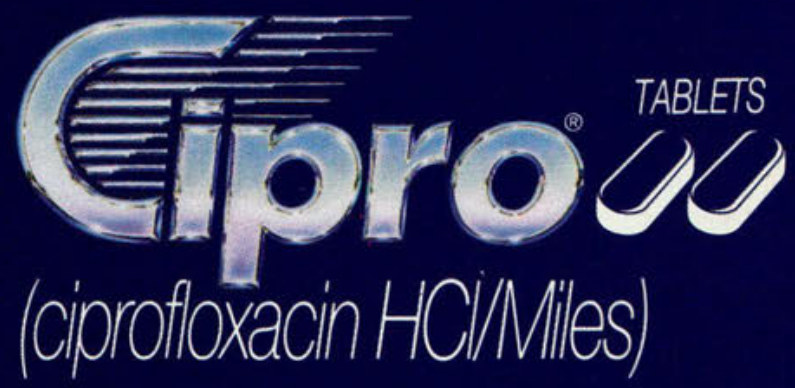

The eradicator:" 


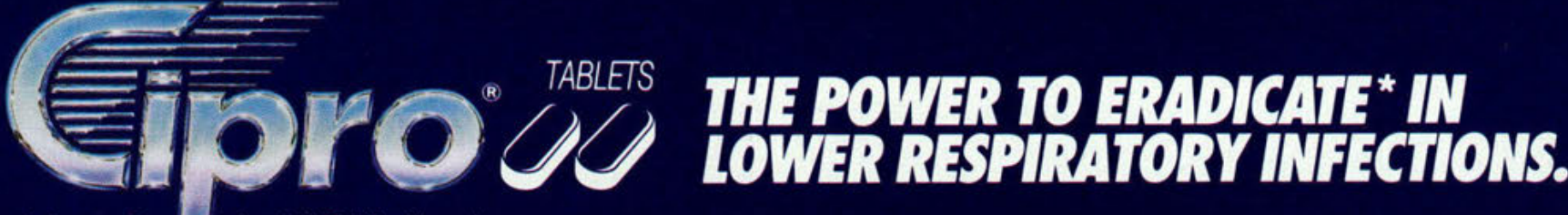 (ciprofoxazain HCIMliles)}

CIPRO* TABLETS

(ciprofloxacin $\mathrm{HCl} / \mathrm{Miles}$ )

BRIEF SUMMARY

CONSULT PACKAGE INSERT FOR FULL PRESCRIBING INFORMATION INDICATIONS AND USAGE

Cipro* is indicated for the treatment of infections caused by susceptible strains of the designated microorganisms in the conditions listed below

Lower Respiratory Inlections caused by Escherichia coli. Klebsiella pneumonae. Enterobacter cloacae. Proteus mirabills. Pseudomonas aeruginosa. Haemophilus influenzae. Haemophilus paramilluenzae, and Streptococcus

Speumoniae. Proteus mirabilis. Proteus vulgaris. Providencia stuartui. Morganella morganiv. Citrobacter freundiu, Pseudomona. aeruginosa. Staphylococcus aureus. Staphylococcus epidermidis, and Streptococcus pyogenes

Bone and Joint Inlections caused by Enterobacter claacae. Serratia marcescens. and Pseudomonas aerugunosa Urinary Iract Intections caused by Eschericha coli, Klebsiella pneumoniae. Enterobacter cloacae. Serratia ma cescens. Proteus mirabilis. Providencia reftgerv. Morganella morganin. Citrobacter diversus. Citrobacter freundit. Pseudomonas aeruginosa. Staphylococcus epidermidis, and Streptococcus faecalis

Intectious Diarnhea caused by Escherichia coli (enterotoxigenic strains). Campylobacter jefunu. Shigella flexnen. and Shigella sonner" when antibacterial therapy is indicated

'Eflicacy for this organism in this organ system was studied in tewer than 10 infections CONTRAINDICATIONS

A history of hypersensitvity to ciprofloxacin is a contraindication to its use. A history of hypersensitivity to other quinolones may also contraindicate the use of ciprofloxacin.

\section{WARNINGS}

CIPROFLOXACIN SHOULD NOT BE USED IN CHILDREN. ADOLESCENTS. OR PREGNANT WOMEN. The oral admin istration of ciprofloxacin caused lameness in immature dogs. Histopathological examination of the weight-bearing joints of these dogs revealed permanent lesions of the cartilage. Related drugs such as nalidixic acid. cinoxacin, and joritloxacin also produced erosions of cartilage of weight-bearing joints and other signs of arthropathy in immature norfloxacin also produced erosions of cartilage of weight-bearing joints and other signs of arthropathy in immana
animals of various species (SEE ANIMAL PHARMACOLOGY SECTION IN FULL PRESCRIBING INFORMATION) PRECAUTIONS

General: As with other quinolones. ciprofloxacin may cause central nervous system (CNS) stimulation, which may lead to tremor, restlessness, lightheadedness. confusion, and rarely to hallucinations or convulsive seizures. There fore, ciprofloxacin should be used with caution in patients with known or suspected CNS disorders. such as sevecerebral arterioscierosis or epilepsy. or other tactors which predispose to seizures (SEE ADVERSE REACTIONS). Anaphylactic reactions following the first dose have been reported in patients receiving therapy with quinolones Some reactions were accompanied by cardiovascular collapse. loss of consciousness, tingling. pharyngeal or tacca edema. dyspnea, urticaria, and itching. Only a few patients had a history of hypersensitivity reaction. Anaphylactic
reactions may require epinephrine and other emergency measures. Ciprofloxacin should be discontinued at the first sign of hypersensitivity or allergy

sign of hypersensitivity or allergy

Severe hypersensitivity reactions characterized by rash, tever, eosinophilia. jaundice, and hepatic necrosis with fatal outcome have been reported rarely (less than one per million prescriptions) in patients receiving ciprofloxacin along with other drugs. The possibility that these reactions were related to ciprotloxacin cannot be excluded Ciprofloxacin should be discontinued at the first appearance of a skin rash or any sign of other hypersensitivity reaction

Crystals of ciprofloxacin have been observed rarely in the urine of human subjects but more frequently in the urine of laboratory animals (SEE ANIMAL PHARMACOLOGY SECTION IN FULL PRESCRIBING INFORMATION Crystalluria related to ciprotloxacin has been reported only rarely in man, because human urine is usually acidic.
Pattients receiving ciprofloxacin should be well hydrated. and aikalinity of the urine should be avoided. The

recommended dally dose should not be exceeded.
Atteration of the dosage regmen is necessary for patients with impairment of renal function (SEE DOSAGE AND Alteration of the
ADMINISTRATION)

As with any potent drug. periodic assessment of organ system functions. including renal, hepatic. and hematopoietic function, is advisable during prolonged therapy

Drug Interactions: As with other quinolones. concurrent administration of ciprofloxacin with theophyline may lead to elevated plasma concentrations of theophylline and prolongation of its elimination haif-life. This may result in increased risk of theophylline-related adverse reactions. If concomitant use cannot be avoided. plasma levels of theophylline should be monitored and dosage adjustments made as appropriate

Quinolones. including ciprofloxacin. have also been shown to interfere with the metabolism of cafteine. This may lead to reduced clearance of catteine and a proiongation of its plasma hait-life

Antacids containing magnesium hydroxide or aluminum hydroxide may intertere with the absorption of ciprotlox

acin resulting in serum and urine levels lower than desired. concurrent administration of these agents with

ciprofloxacin should be avoided

Concomitant administration of the nonsteroidal anti-inflammatory drug fenbuten with a quinolone has been

reported to increase the risk of CNS stimulation and convulsive sezures

Probenecid interferes with the renal tubular secretion of ciprotioxacin and produces an increase in the level of

ciprofloxacin in the serum. This should be considered if patients are receiving both drugs concomitantly

As with other broad-spectrum antibiotics. prolonged use of ciprofloxacin may result in overgrowth of nonsuscept-

ble organisms. Repeated evaluation of the patient's condition and microbial susceptibility testing is essential. superinfection occurs during therapy. appropriate measures should be taken

Intormation for Patients: Patients should be advised that ciprofloxacin may be taken with or without meals. The preferred time of dosing is two hours atter a meal. Patients should also be advised to drink fluids iberally and not take

antacids containing magnesium or aluminum.
Patients should be advised that cprofloxacin may be associated with hypersensitivity reactions, even following a single dose, and to discontinue the drug at the first sign of a skin rash or other allergic reaction

Ciprofloxacin may cause dizziness or lightheadedness: therefore patients should know how they react to this druo before they oper ate an automobile or machinery or engage in activties requiring mental alertness or coordination Patients should be advised that ciprofloxacin may increase the effects of theophylline and caffeine

Carcinogenesis. Mutagenesis. Impairment of Fertility: Eight in vitro mutagenicty tests have been conducted with

ciprofloxacin and the test results are listed below

Salmonella Microsome Test (Negative)

E. coli DNA Repair Assay (Negative)

Mouse Lymphoma Cell Forward Mutation Assay (Positive

Chinese Hamster $V_{79}$ Cell HGPRT Test (Negative)

Syrian Hamster Embryo Cell Transformation Assay (Negative)

Saccharomyces cerevisiae Point Mutation Assay (Negative)

Saccharomyces cerevisiae Mitotic Crossover and Gene Conversion Assay (Negative)

Rat Hepatocyte DNA Repar Assay (Positive)

Thus. two of the eight tests were positive. but the results of the following three in vivo test systems gave negative results

Rat Hepatocyte DNA Repair Assay

Micronucleus Test (Mice)

Long-term carcinogenicity studies in rats and mice have been completed. Atter daily oral dosing for up to 2 years there is no evidence that ciprofloxacin had any carcinogenic or tumorigenic effects in these species

Pregnancy - Pregnancy Category C. Reproduction studies have been performed in rats and mice at doses up to 6 times the usual daliy human dose and have revealed no evidence of impaired fertility or harm to the fetus due to ciprofloxacin. In rabbits, as with most antimicrobial agents. ciprofloxacin $(30$ and $100 \mathrm{mg} / \mathrm{kg}$ orally) produced ciprofloxacin. In rabbits, as with most antimicrobial agents. Ciprotioxacin ( 30 and $100 \mathrm{mg} / \mathrm{kg}$ oraliy) produced
gastrointestinal disturbances resulting in maternal weight loss and an increased incidence of abortion. No terato- genicity was observed at either dose. After intravenous administration, at doses up to $20 \mathrm{mg} / \mathrm{kg}$. no maternal toxicity was produced. and no embryotoxicity or teratogenicity was observed. There are, however, no adequate and well. ARTHROPATHY IN IMMATURE ANIMALS. IT SHOULD NOT BE USED IN PREGNANT WOMEN (SEE WARNINGS) Nursing Mothers: It is not known whether ciprofloxacin is excreted in human milk, however, it is known that ciprotloxacin is excreted in the milk of lactating rats and that other drugs of this class are excreted in human milk. Because of this and because of the potential for serious adverse reactions from ciprofloxacin in nursing infants. a decision should be made to discontinue nursing or to discontinue the drug. takng into account the importance of the drug to the mother

Pediatric Use: Patients under the age of 18 were not included in the clinical trials of ciprofloxacin because ciprotloxacin as well as other quinolones causes arthropathy in immature animals Ciprotloxacin should not be used in children or adolescents (SEE WARNINGS)

\section{ADVERSE REACTIONS}

Ciprofloxacin is generally well tolerated. During clinical investigation. 2.799 patients recerved 2.868 courses of the drug. Adverse events that were considered likely to be drug related occurred in $7.3 \%$ of courses. possibly related in $92 \%$. and remotely related in $3.0 \%$. Ciprofloxacin was discontinued because of an adverse event in $3.5 \%$ of courses.
primarly involving the gastrontestinal system $(1.5 \%)$. skin $(0.6 \%)$. and central nervous system $(0.4 \%)$. Those primarily involving the gastrointestinal ssste

The most frequently reported events, drug related or not, were nausea (5.2\%), diarthea $(2.3 \%)$, vomiting $(2.0 \%)$

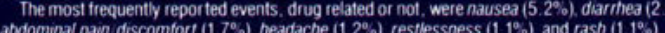

abdominal pain discomfort $(1.7 \%)$, headache $(1.2 \%)$. restlessness $(1.1 \%)$, and rash $(1.1 \%$

GASTROINTESTINAL: (See above), paintul oral mucosa, oral candidiasis, dysphagia. intestinal perforation,

gastrointestinal bleeding. bons. manic reaction, irritability, tremor, ataxia, convulsive seizures, lethargy, drowsiness, weakness. mal. aise, anorexia, phobia, depersonalization, depression, paresthesia
SKIN HYPERSENSITIVITY (See above) pruritus, urticaria. pho

SKIN HYPERSENSITIVITY: (See above), pruritus, urticaria, photosensitivity, flushing, fever, chills angioedema, edema of the face, neck, lips, conjunctivae or hands. cutaneous candidiasis. hyperpigmentation. erythema nodosuin

Allergic reactions ranging from urticaria to anaphylactic reactions have been reported (SEE PRECAUTIONS) SPECIAL SENSES: blurred vision, disturbed vision (change in color perception, overbrightness of lights) decreased visual acuity. diplopia, eve pain, tinnitus, hearing loss, bad taste

MUSCULOSKELETAL: joint or back pain, joint stitfness. achiness. neck or chest pain. flare-up of gout

RENAL UROGENITAL: intersotital nephritis. nephritis. renal tailure. polyuria, urinary retention, urethral bleeding, vaginits, acidosis

CARDIOVASCULAR: paipitations, atrial flutter, ventricular ectopy. syncope, hypertension. angina pectoris. myocardial infarction. cardiopulmonary arrest. cerebral thrombosis

RESPIRATORY: epistaxis, laryngeal or pulmonary edema, hiccough, hemoptysis. dyspnea, bronchospasm, pulmonary embolism

Most of the adverse events reported were described as only mild or moderate in severity. abated soon after the drug was discontinued. and required no treatment

In several instances, nausea, vomiting. tremor, restlessness, agitation, or palpitations were judged by investigators to be related to elevated plasma levels of theophylline possibly as a result of a drug interaction with ciprofloxacin

Other adverse events reported in the postmarketing phase include anaphylactoid reactions. Stevens-Johnson syndrome, exfoliative dermattis, toxic epidermal necrolysis, hepatic necrosis, postural hypotension, possible exacerbation of myasthenia gravis, confusion. dysphasia, nystagmus, pseudomembranous colitis, dyspepsia, cholesterol, blood glucose, serum potassium, prolongation of prothrombin time, albuminuria; candiduria, vagina candidiasis, and renal calculi (SEE PRECAUTIONS

Adverse Laboratory Changes: Changes in laboratory parameters isted as adverse events without regard to drug relationship

Hepatic-Elevations of: ALT (SGPT) (1.9\%). AST (SGOT) (1.7\%), alkaline phosphatase $(0.8 \%)$, LOH $(0.4 \%)$. serum bilirubin $(0.3 \%)$

Cholestatic jaundice has been reported

Hematologic-Eosinophilia $(0.6 \%)$, leukopenia $(0.4 \%)$, decreased blood platelets $(0.1 \%)$, elevated blood platelets $(0.1 \%)$, pancytopenia $(0.1 \%)$

Renal-Elevations of: Serum creatinine (1.1\%). BUN $(0.9 \%)$

CRYSTALLURIA, CYLINDRURIA. AND HEMATURIA HAVE BEEN REPORTED

Other changes occurring in less than $0.1 \%$ of courses were: Elevation of serum gammaglutamyl transferase. bleeding diathesis, increase in blood monocytes, and leukocytosis.

\section{ONERDOSAGE}

Information on overdosage in humans is not available. In the event of acute overdosage. the stomach should be emptied by inducing vomiting or by gastric lavage. The patient should be carefully observed and given supportive treatment. Adequate hydration must be maintained. Only a small amount of ciprofloxacin $(<10 \%)$ is removed from the body after hemodialysis or peritoneal dialysis

\section{DOSAGE AND ADMINISTRATIO:}

The usual adult dosage for patients with urinary tract infections is $250 \mathrm{mg}$ every 12 hours. For patients with complicated infections caused by organisms not highly susceptible. $500 \mathrm{mg}$ may be administered every 12 hours Lower respiratory tract infections, skin and skin structure infections, and bone and joint infections may be treated with $500 \mathrm{mg}$ every 12 hours. For more severe or complicated infections. a dosage of $750 \mathrm{mg}$ may be given every 12 The

he recommended dosage for infectious diarrhea is $500 \mathrm{mg}$ every 12 hours

In patients with renal impairment. some modification of dosage is recommended (SEE DOSAGE AND AD MINISTRATION SECTION IN FULL PRESCRIBING INFORMAAION).
HOW SUPPLIED Cipro* (ciprofloxacin HCI Miles) is avallable as tablets of $250 \mathrm{mg}$. $500 \mathrm{mg}$. and $750 \mathrm{mg}$ in bottles of 50 , and in Unit-
Dose packages of 100 (SEE FULL PRESCRIBING INFORMATION FOR COMPLETE DESCRIPTION).

Reterence: 1. Data on file. Miles Inc Pharmaceutical Division

Achieves $96 \%$ tavorable clinical response (resolution + improve

For further information, contact the Miles Information Service:

1-800-642-4776. In VA, coll colled: 703-391-7888.

COMMITTED TO THERAPEUTIC EFFICIENCY

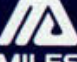

Miles Inc.

400 Morgan Lane

West Haven, CT 06516 
phragm works at maximum efficiency. Shorter fiber lengths, associated with a flattened diaphragm, have decreased forces of contraction. The infant diaphragm in a nondiseased state has a long resting fiber length capable of generating high inspiratory forces of contraction.

The power of diaphragmatic contraction is determined by multiplying the force of contraction by the velocity, where velocity is defined as the actual velocity divided by the resting fiber length. Therefore, for any given lung volume there is a unique combination of force and velocity of contraction that provides maximal power. With changing lung volumes and inspiratory demand, the efficiency of the system is maintained by the addition or subtraction of the accessory muscles of respiration. The accessory muscles include the intercostal, sternocleidomastoid, scalene, and abdominal muscle groups.

These accessory muscle groups are active, even at resting lung volumes. Because of the lung's poor compliance at birth, most of the effort of the newborn's intercostal muscles is directed toward fixation, not expansion of the thoracic cage. Following the newborn period, the intercostal muscles are used to alter thoracic volume. The previously held notion that the external intercostal muscles effected inspiration and the internal intercostal muscles effected expiration appears to be an oversimplification. Recent studies have demonstrated that both sets of intercostal muscles are active in varying degrees throughout the different phases of inspiration and expiration.

The scalene muscles appear to stabilize the chest at rest and actively augment thoracic expansion in the later phases of inspiration. The sternocleidomastoid muscles are the only true accessory muscles of respiration because they are active only during periods of significantly increased respiratory work loads. The abdominal musculature plays a small, but significant, part in stabilizing the abdominal cavity. This allows for increased thoracic expansion with diaphragmatic contraction.

\section{Specific pharmacologic agents Methylxanthines}

The methylxanthines theophylline and caf- feine produce a wide spectrum of pharmacologic actions. Despite the widespread use of theophylline in adult and pediatric populations, the precise mechanisms and actions of this drug have not been fully clarified.

Theophylline has several proposed mechanisms of action with regard to respiratory control and function. The most widely accepted of these is the proposition that cyclic adenosine monophosphate (cAMP) is increased by the inhibition of phosphodiesterase. Conversely, it is argued that the first-order kinetics that regulate cAMP production do not account for the changes seen in cAMP relative to changes in theophylline. In addition, other xanthines that inhibit phosphodiesterase do not significantly alter bronchial smooth-muscle tension. A proposed alternative explanation is that theophylline acts as an analogue to cAMP. However, it has been demonstrated that smooth muscle may relax without changes in cAMP.

The interaction between theophylline and adenosine is complex and incompletely understood. This putative, or contributory, mechanism may help explain both the peripheral and centrally mediated effects. Adenosine receptor inhibition may allow an increased transmembrane flux of calcium, causing increased muscle contractility and resistance to fatigue. Theophylline enhances skeletal muscle contractility and inhibits fatigue. Calcium channel blockers can inhibit theophylline's positive effects on diaphragmatic contractility. Nonadenosine-antagonist xanthine derivatives do not have this same effect.

Adenosine has been shown to be a central nervous system depressant in some animals, although this effect has never been proved in human infants. Theophylline antagonism of adenosine receptors may account for the central stimulation of theophylline. This depressant effect is increased in the younger animal model and may account for the differences in the degree of theophylline-induced central stimulation. The induced elevation of catecholamine and its possible role as a prostaglandin antagonist may contribute to theophylline's central action.

In infants, theophylline has been widely 
used in the treatment of bronchopulmonary dysplasia (BPD) and apnea. Its success in the treatment of BPD has been attributed to the reduction of airway resistance and subsequent improvement in lung function, rather than to theophylline's effects on respiratory drive.

The efficacy of theophylline and caffeine in infant apnea is well documented. Observations that the reduction of apnea and periodic breathing occurs without significant alterations in lung function support the concept of a centrally mediated process. Conversely, in the premature infant, apnea may be secondary to hypoventilation that is a function of a highly compliant chest wall, muscle fatigue, and premature lung dynamics. All these factors are affected by methylxanthines. Adequate measurement and control of these factors require sensitive, sophisticated instruments and methods, which are not always available.

Some medical centers prefer caffeine to theophylline for the treatment of idiopathic apnea. One double-blind study of caffeine and theophylline demonstrated equivalent efficacy in the treatment of apnea of prematurity. ${ }^{1}$ Caffeine has the additional benefits of a wider range of safety, single daily-dose administration, and a more consistent steady-state status.

Theophylline has been proposed as a method of enhancing the respiratory pump by improving diaphragmatic contractility and resistance to fatigue; however, there has been significant controversy concerning the accuracy of this ap eeeproach in vivo. Most animal studies have shown that the concentration of theophylline required to achieve significant improvement of contractility in vitro is in the potentially toxic range for humans. This conclusion, however, may be related to study design. The in vitro experiments that required high doses of theophylline also involved diminished blood supply to the muscle tissue. In addition, the high levels of theophylline in circulating media may not correlate on a one-to-one basis with in vivo plasma levels. Studies in both healthy adults and in adults with obstructive lung disease, have shown a small but significant improvement in diaphragmatic contractility in fatigue and nonfatigue conditions.

Although the differences in fiber composi- tion between infant and adult diaphragms may suggest a limited applicability of the adult studies in infant medicine, a recent study demonstrates a similar diaphragmatic response to theophylline in adults and infants. In a study of 18 infants of less than 31 weeks' gestation with resolving respiratory distress syndrome (RDS), aminophylline caused a $68 \%$ increase in diaphragmatic excursion as measured by ultrasound. ${ }^{2}$ The clinical significance of this improved diaphragmatic contractility has not been established but can be inferred to possibly aid in syndromes where small tidal volumes and increased work loads are pathologic entities.

Theophylline is used in some medical centers for aiding extubation from mechanical ventilation. The efficacy of this use has not been well established in the literature. One study ${ }^{3}$ examined the use of theophylline in BPD and found efficacy only in a small group of infants younger than 1 month. In two subsequent studies, ${ }^{4,5}$ infant patients receiving this drug were examined in the weaning phase of the RDS. Both of these latter studies suffer from small sample size and poor study design, prohibiting an accurate assessment of the clinical efficacy of the drug.

Clinically, theophylline is commonly used in two situations: apnea and moderate to severe BPD. The dose varies for each of these indications. The respiratory stimulation effect is thought to occur at lower levels than is needed for bronchodilation. Desired serum levels for respiratory stimulation range between $6 \mathrm{mg} / \mathrm{dL}$ and $12 \mathrm{mg} / \mathrm{dL}$. Desired serum levels for bronchodilation and treatment of BPD range from $10 \mathrm{mg} / \mathrm{dL}$ to $20 \mathrm{mg} / \mathrm{dL}$. By using a volume of distribution of $0.5 \mathrm{~L} / \mathrm{kg}$ (range, 0.3 $0.7 \mathrm{~L} / \mathrm{kg}$ ), one can calculate a loading dose by the following equation:

$$
\text { desired serum level }=\frac{\text { dose }}{\text { volume of distribution }}
$$

There are various recommendations for maintenance dosing. A popular one for apnea is $2 \mathrm{mg} / \mathrm{kg} /$ dose every 8 hours. Popular ones for bronchodilation are $2 \mathrm{mg} / \mathrm{kg} /$ dose every 6 hours for infants 0 to 3 months of age, or the 
following total daily dose for infants older than 3 months:

\section{$[0.3 \times$ age in weeks $]+8$ per day}

All dosing schedules need to be adjusted using actual serum levels as a guide. By convention, trough levels 1 hour prior to administration are obtained for apnea and peak levels from 1 to 4 hours after administration (depending on the theophylline type used) are obtained for bronchodilation. These are times at which steady state presumably has been achieved.

Caffeine is primarily used in the treatment of apnea syndromes, although there is growing support for its efficacy as an adjuvant in the treatment of moderate to severe BPD. The desired serum concentration in the treatment of apnea is $5 \mu \mathrm{g} / \mathrm{mL}$ to $30 \mu \mathrm{g} / \mathrm{mL}$. Usually, toxic side effects are not apparent until levels exceed $50 \mu \mathrm{g} / \mathrm{mL}$. A loading dose of $10 \mathrm{mg} / \mathrm{kg}$ (anhydrous base) is followed in 12 to 24 hours by a total daily dose of $2.5 \mathrm{mg} / \mathrm{kg}$. The maintenance dose may be given once a day while still achieving an adequate plasma steady state. Monitoring of the steady-state level begins after administration of the fifth maintenance dose. Theophylline is metabolized to caffeine. When converting from theophylline to caffeine therapy, a reasonable approach is to initiate a half loading bolus, followed by the usual maintenance dose.

\section{Doxapram}

Doxapram is one of the most potent and least toxic respiratory stimulants used in adults. Its primary mode of action is through the stimulation of peripheral chemoreceptors. At high doses, the medullary center is also affected. In cats with ablated efferent peripheral chemoreceptor pathways, there is ventilatory stimulation at high doses but not at low doses. In human infants, administration of doxapram at low doses results in a significant increase in minute ventilation. This is primarily a result of an increased tidal volume rather than increased respiratory rate. ${ }^{6}$

Doxapram is used by several centers to treat apnea of prematurity refractory to xanthines. It has not been as intensively studied as the xanthines; therefore, at the present time it is not considered a first-line agent for the treatment of neonatal apnea. In addition, the lack of an oral preparation makes it less appealing than the xanthines for clinical use. Although studies currently supporting doxapram's use have uniformly agreed on its efficacy, these studies are fraught with design weaknesses that do not permit an unequivocal determination of doxapram's role in the treatment of apnea syndromes..$^{7-9}$

Doxapram has also been investigated for its use in weaning RDS infants from the ventilator. In one small study, ${ }^{5}$ statistical difference was not achieved.

Hunt and colleagues ${ }^{10}$ demonstrated clinically significant improvement with use of doxapram in the treatment of congenital hypoventilation syndrome. However, their study involved a small sample size, and they reported an unusually large number of side effects that may have been dose related. The dose used was two to three times the dose administered in apnea trials. Given the complex nature of congenital hypoventilation syndrome, it is unclear whether the clinical efficacy would be retained at the lower, safer doses, which do not affect medullary centers.

Reported side effects are primarily a function of increased neurogenic and adrenergic output. They include irritability, hyperactivity, hyperthermia, hypertension, and possibly seizures. These side effects are rare and occur most frequently in association with doses greater than $2.5 \mathrm{mg} / \mathrm{kg} / \mathrm{hr}$ or when doxapram is used in conjunction with theophylline. In the apnea studies in which doxapram was administered as a single agent and the dose was less than $3 \mathrm{mg} / \mathrm{kg} / \mathrm{hr}$, no adverse effects were noted. ${ }^{6-8}$

Various formulations of doxapram are available. Although an oral preparation is available in England, only the parenteral route is approved in the United States. In Canada, a $0.5 \%$ solution of chlorobutanol is used as the preservative. In the United States, a $0.9 \%$ solution of benzyl alcohol is the preservative used. The potentially toxic level of benzyl alcohol in the neonate has been retrospectively estimated at $99 \mathrm{mg} / \mathrm{kg} / \mathrm{d}$. Recently, the use of 
benzyl alcohol at any dose in the neonate has been questioned.

In a retrospective analysis, ${ }^{11}$ the use of flush with benzyl alcohol as a bacteriostatic agent was significantly associated with the development of kernicterus and intraventricular hemorrhage. The amount of benzyl alcohol infused was not specified, but it can be estimated from the data reported to have been at a minimum of $150 \mathrm{mg} / \mathrm{kg} / \mathrm{d}$. Doxapram, if infused at a dose of $2.0 \mathrm{mg} / \mathrm{kg} / \mathrm{hr}$, will deliver $22 \mathrm{mg} / \mathrm{kg} / \mathrm{d}$ of benzyl alcohol. This is $15 \%$ to $20 \%$ of the retrospectively determined toxic dose. In comparison, phenobarbital delivers $2 \mathrm{mg} / \mathrm{kg} / \mathrm{d}$ of benzyl alcohol and pancuronium bromide delivers $1 \mathrm{mg} / \mathrm{kg} / \mathrm{d}$.

Only one study has been performed using doxapram with benzyl alcohol for the treatment of apnea. ${ }^{12}$ Significant improvement in ventilation was achieved in 22 infants with apnea refractory to theophylline without any evidence of an increased risk of intraventricular hemorrhage.

Doxapram is a potentially viable, but temporary, therapy in the treatment of refractory apnea and congenital hypoventilation syndromes. The respiratory-stimulating effects are dose dependent and vary among individuals. A reasonable schedule would be a beginning infusion of $0.5 \mathrm{mg} / \mathrm{kg} / \mathrm{hr}$ subsequently increased by $0.5-\mathrm{mg} / \mathrm{kg} / \mathrm{hr}$ increments until clinical improvement or a maximum level of 2.5 $\mathrm{mg} / \mathrm{kg} / \mathrm{hr}$ is reached. Doses in excess of this level continue to have an increasing stimulating action, but the risk of side effects may be inhibitory.

\section{Naloxone}

Exogenous and endogenous endorphins/opiates are central respiratory center depressants through their direct interaction with opiate receptors in the medulla. Part of the rationale for using endorphin antagonists as respiratory modulators has come from the use of naloxone as an endorphin antagonist. The administration of naloxone in a number of animal studies $^{13-15}$ resulted in an increased ventilatory drive, especially in the presence of hypoxia. This effect is inversely related to age and appears to be most significant during the fetal stage and the immediate perinatal period.

In asphyxiated neonates, there is an increased presence of endorphins, which may be associated with a depressed respiratory status and diminished total body demand for oxygen. ${ }^{14-17}$ Unlike animal studies, studies of human infants have not demonstrated the degree of significant reversal of endorphin-induced respiratory depression by naloxone. ${ }^{16,18,19} \mathrm{~A}$ study of 11 healthy newborn infants exposed to $15 \%$ $\mathrm{O}_{2}$ for 5 minutes found a $14 \%$ decrease of minute ventilation following saline injection but only a $4 \%$ decrease following naloxone injection. ${ }^{18}$ The differences in minute ventilation were related to tidal volume and not to respiratory rate.

Multiple animal trials have demonstrated the usefulness of naloxone in the reduction of respiratory depression following perinatal asphyxia. Nevertheless, a 1988 study of human infants ${ }^{19}$ did not demonstrate clinical efficacy for naloxone in either the severely asphyxiated or moderately asphyxiated groups. ${ }^{19}$

In many of the studies, including the aforementioned human neonate studies, the researchers used doses that frequently ranged from 10 to 40 times higher than the recommended dose for reversal of narcotic-induced respiratory depression. Alhough naloxone is commonly viewed as a specific endorphin/opiate antagonist, this may not necessarily be entirely accurate. At higher doses, the action of naloxone may not be specific and may involve other neuromodulators in addition to endorphins.

Whether naloxone is related to endorphins or functions by another mechanism, its usefulness as a clinical agent for the reversal of endogenous opiate-induced respiratory depression remains unproved. At this time, there is no evidence for the clinical efficacy of naloxone in augmenting the respiratory drive during conditions of respiratory depression except in the presence of exogenous narcotics.

The dose recommended for the reversal of narcotic-induced respiratory depression is 0.01 $\mathrm{mg} / \mathrm{kg}$. This dose may be repeated several times. Side effects at this and larger doses are extremely uncommon. There have been reports of altered hemodynamic status, cardiac 
dysrhythmias, and neurogenic stimulation. It is unclear whether these events are directly related to the narcotic reversal, are part of a postanesthetic state, or are a direct consequence of the naloxone.

\section{Almitrine bismesylate}

Almitrine bismesylate has been administered to adults with chronic obstructive pulmonary disease (COPD) to improve their ventilatory status. The increase in $\mathrm{PaO}_{2}$ and the decrease in $\mathrm{PaCO}_{2}$ may be due to alterations of the respiratory control mechanism, pulmonary vasculature, or a combination of the two.

In both healthy adults and adults with COPD, almitrine alters the respiratory dynamics by improving tidal volume, increasing flow rate, and decreasing the inspiratory-to-expiratory ratio. However, many studies indicate that the blood gas changes are primarily due to pulmonary vasculature alterations improving the existing ventilatory-perfusion mismatch in COPD patients.

The use of almitrine in infants has been limited to the treatment of congenital hypoventilation syndromes. There have been a few reports of cases in which there was improvement, but a fairly well-controlled study of 12 children did not demonstrate statistical or clinical improvement of ventilatory or gas exchange parameters. ${ }^{20,21}$

The results of the study by Oren and associates ${ }^{21}$ do not preclude the use of almitrine for improving ventilation in other disease states. Congenital hypoventilation syndrome is most likely a spectrum of disease states reflecting multilevel dysfunction of the respiratory center at both the peripheral and central levels. In a heterogeneous, nonstratified population, the efficacy of almitrine may not be identified. Also, no attempt has been made to duplicate the experience in adult patients with COPD in the BPD population, a subset of infants with obstructive lung disease and altered pulmonary artery hemodynamics.

Almitrine may also prove useful in resetting the peripheral chemoreceptor hypoxic drive in the immediate postnatal period. In a single study of lambs, ${ }^{22}$ almitrine was successful in augmenting the resetting of the hypoxic drive. The use of almitrine in this situation has not been fully evaluated in other animal models or humans.

\section{Progesterone}

Progesterones increase the respiratory drive of both men and women when they are healthy and in a state of pulmonary embarrassment. It is postulated that the mechanism is centrally mediated either directly or through metabolites. In the resting state, the increased minute ventilation is from a larger tidal volume and not from an increase in respiratory rate, metabolism, or mechanical factors. The ventilatory pattern is augmented in response to a work load by increasing both chemoreceptor and neuromechanoreceptor activity. ${ }^{23-25}$ There are only isolated reports of the use of progesterone in infants. The role of progesterone in the modulation of the respiratory drive in the intrauterine and extrauterine environment remains unproved. An imbalance of progesterone may play a role in transitory hypoventilation or tachypneic states during the period shortly after birth.

\section{Prostaglandins}

During the initial studies ${ }^{26,27}$ of the use of prostaglandin $\mathrm{E}_{1}\left(\mathrm{PGE}_{1}\right)$ to maintain ductal patency, respiratory depression was an occasional complication. In one large study, the incidence was $12 \%$. Conversely, there appears to be an improvement in $\mathrm{PaCO}_{2}$ with the use of indomethacin, independent of ductal closure. This is postulated to be a direct effect of improved alveolar ventilation through the decreased production of E-type prostaglandins (PGEs), which appear to be central respiratory drive inhibitors. ${ }^{26,27}$

Numerous animal studies have demonstrated that PGEs have a direct inhibitory effect on the central respiratory center. Reduction of $\mathrm{PGE}_{2}$ levels at the time of birth is related to, but not a sole determinant of, the onset of neonatal breathing. The administration of PGEs results in ventilatory depression. ${ }^{28,29}$

The use of prostaglandin synthetase inhibitors uniformly results in lowered levels of $\mathrm{PaCO}_{2}$. This may be a ventilation effect from the lowered levels of PGEs and a subsequent 
diminished inhibition of the central respiratory drive. There also may be a perfusion effect from vasodilation contributing to the lowered $\mathrm{PaCO}_{2} \cdot{ }^{28,30}$

Currently there are no clinical indications for the use of prostaglandins or their inhibitors for the manipulation of the respiratory drive.

\section{Conclusion}

The infant's respiratory control center and pump mechanism are frequently insufficient to provide adequate ventilation in states of health or illness. With the exception of its use for apnea and for the reversal of opiate-induced respiratory depression, pharmacologic manipulation as a means of significantly altering the outcome of an imbalanced, insufficient respiratory center and pump mechanism remains unproved, but is theoretically promising. Its usefulness invites further investigation.

1. Bairam A, Boutroy MJ, Badonnel Y, et al: Theophylline versus caffeine: Comparative effects in the treatment of idiopathic apnea in the preterm infant. J Pediatr 1987;110:636-639.

2. Heyman E, et al: Apnea in preterm infants: Role of aminophylline and diaphragmatic contractility. Abstract 1862, Society for Pediatric Research, 1989.

3. Rooklin AR, Moomjian AS, Shutack JG, et al: Theophylline therapy in bronchopulmonary dysplasia. JPediatr 1979;95:882885.

4. Harris MC, Baumgart S, Rooklin AR, et al: Successful extubation of infants with respiratory distress syndrome using aminophylline. J Pediatr 1983;103:303-305.

5. Eyal FG, Sagi EF, Alpan G, et al: Aminophylline versus doxapram in weaning premature infants from mechanical ventilation: Preliminary report. Crit Care Med 1985;13:124-125.

6. Barrington KJ, Finer NN, Peters KL, et al: Physiologic effects of doxapram in idiopathic apnea of prematurity. J Pediatr 1986;108:125-129.

7. Hayakawa F, Susumu H, Kuno K, et al: Doxapram in the treatment of idiopathic apnea of prematurity: Desirable dosage and serum concentrations. $J$ Pediatr 1986;109:138-140.

8. Barrington KJ, Finer NN, Torok-Both G, et al: Dose-response relationship of doxapram in the therapy for refractory idiopathic apnea of prematurity. Pediatrics 1987;80:22-27.

9. Eyal F, Alpan G, Sagi E, et al: Aminophylline versus doxapram in idiopathic apnea of prematurity: A double-blind controlled study. Pediatrics 1985;75:709-713.

10. Hunt CE, Inwood RJ, Shannon DC: Respiratory and nonrespiratory effects of doxapram in congenital central hypoventilation syndrome. Am Rev Respir Dis 1979;199:263-269.

11. Jardine DS, Rogers K: Relationship of benzyl alcohol to ker- nicterus, intraventricular hemorrhage, and mortality in preterm infants. Pediatrics 1989;83:153-160.

12. Cartaya E, et al: Efficacy and safety of doxapram made in USA in the therapy of apnea of prematurity refractory to methylxanthine treatment. Abstract 1812, Society for Pediatric Research, 1989.

13. Chernick V, Madansky DL, Lawson EE: Naloxone decreases the duration of primary apnea with neonatal asphyxia. Pediatr Res 1980;14:357-359.

14. Grunstein MM, Hazinski TA, Schlueter MA: Respiratory control during hypoxia in newborn rabbits: Implied action of endorphins. J Appl Physiol 1981;51:122-130.

15. Joseph SA, McMillen IC, Walker DW: Effects of naloxone on breathing movements during hypercapnea in the fetal lamb. $J$ Apple Physiol 1987;62:673-678.

16. Wardlaw SL, Stark RI, Baxi L, et al: Plasma $\beta$-endorphins and $\beta$-lipotropin in the human fetus at delivery: Correlation with arterial $\mathrm{pH}$ and $\mathrm{PO}_{2} . J$ Clin Endocrinol Metab 1979;49:888891.

17. Fleetham JA, Clarke H, Dhingra S, et al: Endogenous opiates and chemical control of breathing in humans. Am Rev Respir Dis 1980;121:1045-1049.

18. De Boeck C, Van Reempts $P$, Rigatto $H$, et al: Naloxone reduces decreases in ventilation induced by hypoxia in newborn infants. $J$ Appl Physiol 1984;56:1507-1511.

19. Chernick V, Manfreda J, De Booy V, et al: Clinical trial of naloxone in birth asphyxia. J Pediatr 1988;113:519-525.

20. Fleming PJ, Levine MR, Lewis GT, et al: Almitrine bismesylate in congenital central hypoventilation. Eur $J$ Respir Dis 1983;126(suppl):307-312.

21. Oren J, Newth CJ, Hunt CE, et al: Ventilatory effects of almitrine bismesylate in congenital central hypoventilation syndrome. Am Rev Respir Dis 1986;134:917-919.

22. Blanco CE, Hanson MA, McCooke HB: Effects of almitrine bismesylate on chemoreceptor activity in fetal sheep and newborn lambs. Eur J Respir Dis 1983;64(suppl 126):313.

23. Kimura H, Hayashi F, Yoshida A, et al: Augmentation of $\mathrm{CO}_{2}$ drives by chlormadinone acetate, a synthetic progesterone. J Appl Physiol 1984;56:1627-1632.

24. Skatrud JB, Dempsey JA, Kaiser DG: Ventilatory response to medroxyprogesterone acetate in normal subjects: Time course and mechanism. J Appl Physiol 1978;44:393-344.

25. Rajagopal K, Abbrecht P, Jabbari B: Effects of medroxyprogesterone acetate in obstructive sleep apnea. Chest 1986;90:815

26. Halliday HL, Hirata T, Brady JP: Indomethacin therapy for large patent ductus arteriosus in the very low birth weight infant: Results and complications. Pediatrics 1979;64:154-159.

27. Lewis AB, Freed MD, Heymann MA, et al: Side effects of therapy with prostaglandin $\mathrm{E}_{1}$ in infants with critical congenital heart disease. Circulation 1981;64:893-898.

28. Kitterman JA: Arachidonic acid metabolites and control of breathing in the fetus and newborn. Semin Perinatol 1987;11:4352.

29. Jansen $\mathrm{AH}$, De Boeck $\mathrm{C}$, Ioffe $\mathrm{S}$, et al: Indomethacin-induced fetal breathing: Mechanism and site of action. $J$ Appl Physiol 1984;57:360-365.

30. Carlson LA, Ekelund LG, Oro L: Circulatory and respiratory effects of different doses of prostaglandin $\mathrm{E}_{1}$ in man. Acta Physiol Scand 1969;75:161-169. 